\title{
ON THE BUSEMANN-PETTY PROBLEM CONCERNING CENTRAL SECTIONS OF CENTRALLY SYMMETRIC CONVEX BODIES
}

\author{
R. J. GARDNER
}

\begin{abstract}
We present a method which shows that in $\mathbb{E}^{3}$ the Busemann-Petty problem, concerning central sections of centrally symmetric convex bodies, has a positive answer. Together with other results, this settles the problem in each dimension.
\end{abstract}

In [BP], Busemann and Petty asked the following question, which resulted from reformulating a problem in Minkowskian geometry. Suppose $K_{1}$ and $K_{2}$ are convex bodies in $n$-dimensional Euclidean space $\mathbb{E}^{n}$ which are centered (centrally symmetric with center at the origin) and such that

$$
\lambda_{n-1}\left(K_{1} \cap u^{\perp}\right) \leq \lambda_{n-1}\left(K_{2} \cap u^{\perp}\right),
$$

for all $u$ in the unit sphere $S^{n-1}$. Then is it true that

$$
\lambda_{n}\left(K_{1}\right) \leq \lambda_{n}\left(K_{2}\right) ?
$$

(Here $u^{\perp}$ denotes the hyperplane through the origin orthogonal to $u$, and $\lambda_{k}$ denotes $k$-dimensional Lebesgue measure, which we identify throughout with $k$-dimensional Hausdorff measure.)

The question, now generally known as the Busemann-Petty problem, has often appeared in the literature. More than thirty years ago, Busemann gave the problem wide exposure in [B2] and Klee posed it again in [K]. The problem attracted the attention of those working in the local theory of Banach spaces; see, for example, the paper [MP, p. 99] of Milman and Pajor. It surfaces again in Berger's article [Be, p. 663], and it is also stated in the books of Burago and Zalgaller [BZ, p. 154]; Croft, Falconer, and Guy [CFG, Problem A9, p. 22]; and Schneider [S, p. 423].

The problem has an interesting history. Using a clever probabilistic argument, Larman and Rogers [LR] proved that the answer, surprisingly, is negative in $\mathbb{E}^{n}$ for $n \geq 12$. Later, Ball [B] applied his work on maximal sections of a cube to obtain a negative answer for $n \geq 10$, where $K_{1}$ is a centered cube and $K_{2}$ a centered ball of suitable radius. Giannapoulos [Gi] improved this negative result to $n \geq 7$ by using an appropriate cylinder for $K_{1}$ instead of a cube. Independently, Bourgain [Bo] showed that the same result can be achieved by taking $K_{1}$ to be a suitable arbitrarily small perturbation of a centered ball;

Received by the editors May 11, 1993.

1991 Mathematics Subject Classification. Primary 52A40; Secondary 28A75, 44A12, 52A15, 52A20, 52A30, 52A38, 92C55.

Key words and phrases. Busemann-Petty problem, convex body, star body, section, intersection body, spherical Radon transform, Schwarz symmetral, geometric tomography.

Supported in part by NSF Grant DMS 9201508. 
Bourgain also proved that his method will not work in $\mathbb{E}^{3}$. A further improvement was made by Papadimitrakis [P] and the author [G1], independently, by demonstrating that the answer is negative for $n \geq 5$, when $K_{1}$ is a centered cylinder. The most recent negative answer was obtained, for $n \geq 4$ and $K_{1}$ a centered cube, by Zhang [Z1], [Z2]. For $4 \leq n \leq 6$, the existence of a suitable $K_{2}$ can be proved, though it seems likely that for these values of $n, K_{2}$ cannot be a ball, regardless of the choice of $K_{1}$. Other papers on the problem related to those mentioned above include [GR] and [T].

We shall outline a solution for $n=3$, thereby settling the problem in each dimension. Against the background of the results above, the positive answer for $n=3$ is unexpected. It is also especially interesting from the point of view of geometric tomography, in which one attempts to obtain information about a geometric object from data concerning its sections or projections. Geometric tomography has connections with functional analysis and possible applications to robot vision and stereology (see, for example, [BL, ES, GV, MP, W]).

A few positive results are already known. The case $n=2$ is trivial. Busemann and Petty themselves noted in [BP] that the Busemann intersection inequality (see [B1, (4), p. 2]) may be applied to obtain a positive answer when $K_{1}$ is a centered ellipsoid. Lutwak [L] obtained an important generalization of this fact by showing that the same is true whenever $K_{1}$ is a member of a certain class of bodies which he called intersection bodies.

We shall explain Lutwak's result in some detail, since it is an essential ingredient in our method. A set $L$ in $\mathbb{E}^{n}$ is star shaped at the origin if it contains the origin and if every line through the origin meets $L$ in a (possibly degenerate) line segment. By a star body we mean a compact set $L$ which is star shaped at the origin and whose radial function

$$
\rho_{L}(u)=\max \{c \geq 0: c u \in L\},
$$

for $u \in S^{n-1}$, is continuous on $S^{n-1}$. The star body $L$ is called the intersection body of another star body $M$ if

$$
\rho_{L}(u)=\lambda_{n-1}\left(M \cap u^{\perp}\right),
$$

for all $u \in S^{n-1}$. We write $L=I M$; it is clear that $L$ must be centered, and it is known (see [L]) that there is a unique centered star body $M^{\prime}$ for which $L=I M^{\prime}$.

A useful alternative viewpoint is provided by the spherical Radon transform. Suppose $g$ is a Borel function on $S^{n-1}$ and $f$ is defined by

$$
f(u)=\int_{S^{n-1} \cap u^{\perp}} g(v) d \lambda_{n-2}(v),
$$

for all $u \in S^{n-1}$; that is, $f(u)$ is the integral of $g$ over the great sphere in $S^{n-1}$ orthogonal to $u$. Then we write

$$
f=R g
$$

and say that $f$ is the spherical Radon transform of $g$. Using the polar coordinate formula for volume, we see that a star body $L$ is the intersection body of some star body $M$ if and only if $\rho_{L}=R g$ for some nonnegative continuous function $g$; just take $g=\rho_{M}^{n-1} /(n-1)$. 
Suppose $L_{1}$ is the intersection body of some star body and $L_{2}$ is an arbitrary star body, such that

$$
\lambda_{n-1}\left(L_{1} \cap u^{\perp}\right) \leq \lambda_{n-1}\left(L_{2} \cap u^{\perp}\right)
$$

for all $u \in S^{n-1}$. Then Lutwak's theorem (see [L, Theorem 10.1]) says that $\lambda_{n}\left(L_{1}\right) \leq \lambda_{n}\left(L_{2}\right)$.

It is worth noting that Lutwak has offered as an alternative and different definition of intersection body, a star body $L$ such that $\rho_{L}=R \mu$, where $\mu$ is an even finite Borel measure in $S^{n-1}$. A consequence of a result in [GLW] is that Lutwak's theorem still holds when $L_{1}$ is an intersection body in this wider sense of the term.

The class of intersection bodies is in a sense dual to the better-known class of projection bodies. The latter, which are just the centered zonoids, have been intensively studied and have many applications; see, for example, the articles of Bourgain and Lindenstrauss [BL], Goodey and Weil [GW], and Schneider and Weil [SW], or Schneider's book [S, Section 3.5]. In fact, the Busemann-Petty problem has a dual form in which sections are replaced by projections. This dual problem was solved, by Petty and Schneider independently, shortly after it was posed; the answer is negative for all $n>2$. Lutwak's theorem concerning sections of star bodies is also dual to a corresponding one for projections of convex bodies, obtained by Petty and Schneider using tools from the BrunnMinkowski theory (see [S, p. 422]). For sections, the extension from convex bodies to star bodies is not only natural but crucial. For example, it can be seen by direct calculation that a centered cylinder in $\mathbb{E}^{3}$ is the intersection body of a nonconvex centered star body; see [G1, Remark 5.2(ii)].

The other known positive results are as follows. Hadwiger $[\mathrm{H}]$ and Giertz [Gie] independently showed that the question has an affirmative answer when $K_{1}$ and $K_{2}$ are coaxial centered convex bodies of revolution in $\mathbb{E}^{3}$. In [G1, Theorem 5.1], it is proved that a centered convex body of revolution $K$ whose radial function $\rho_{K}$ belongs to $C_{e}^{\infty}\left(S^{n-1}\right)$, the class of infinitely differentiable even functions on the unit sphere, is the intersection body of some star body when $n=3$ or 4 . (This result is re-proved in [Z2] by a different method.) Using Lutwak's theorem, it is easy to see that this implies that the BusemannPetty problem has a positive answer in $\mathbb{E}^{3}$ or $\mathbb{E}^{4}$ whenever $K_{1}$ is a centered convex body of revolution. It has also been shown by Meyer [M] that the answer is positive in $\mathbb{E}^{n}$ provided that $K_{1}$ is a centered cross-polytope (the $n$-dimensional version of the octahedron).

We now sketch a proof that the Busemann-Petty problem has a positive answer in $\mathbb{E}^{3}$. The details will appear in [G2].

Theorem. The Busemann-Petty problem has an affirmative answer in $\mathbb{E}^{3}$.

Sketch of the proof. By approximating, we can assume that $\rho_{K_{1}} \in C_{e}^{\infty}\left(S^{2}\right)$ and that $K_{1}$ has everywhere positive Gaussian curvature. By Lutwak's theorem, it suffices to show that an arbitrary centered convex body $K$ in $\mathbb{E}^{3}$ with these additional properties is the intersection body of some star body. (We make no attempt in this note to find the least restrictive additional conditions to impose on $K$.) This will be proved if there is a nonnegative function $g \in C\left(S^{2}\right)$ such that $\rho_{K}=R g$. It is known that since $\rho_{K} \in C_{e}^{\infty}\left(S^{2}\right)$, a $g \in C_{e}^{\infty}\left(S^{2}\right)$ exists and 
is unique. Let $u_{0} \in S^{2}$. An inversion formula of Funk [F] gives

$$
g\left(u_{0}\right)=\lim _{t \rightarrow 1-} \frac{1}{2 \pi} \frac{d}{d t} \int_{0}^{t} \frac{x A_{K}\left(\sin ^{-1} x\right)}{\sqrt{t^{2}-x^{2}}} d x,
$$

where $A_{K}(\phi)$ denotes the average of $\rho_{K}$ on the circle of latitude with angle $\phi$ from the north pole $u_{0}$. After some manipulation, one can obtain

$$
2 \pi g\left(u_{0}\right)=\rho_{K}\left(u_{0}\right)+\frac{1}{2 \pi} \int_{0}^{2 \pi} \int_{0}^{\frac{\pi}{2}} \frac{\partial \rho_{K}(\theta, \phi)}{\partial \phi} \sec \phi d \phi d \theta,
$$

where $(\theta, \phi)$ denotes the usual angles of spherical polar coordinates.

From $K$, construct a body $\bar{K}$, called a Schwarz symmetral of $K$, as follows. Each horizontal section of $\bar{K}$ is a disk whose center lies on the $z$-axis and whose area equals that of the horizontal section of $K$ of the same height. From the Brunn-Minkowski theorem, it follows that $K$ is a convex body of revolution, and our assumptions about $K$ allow us to conclude that $\rho_{R} \in C_{e}^{1}\left(S^{2}\right)$. One can then show that $\rho_{K}=R \bar{g}$, for some $\bar{g} \in C\left(S^{2}\right)$, and that equation (1) holds when $g$ and $\rho_{K}$ are replaced by $\bar{g}$ and $\rho_{K}$, respectively. Moreover, the argument of [G1, Theorem 5.1] proves that $\bar{g}$ is nonnegative.

The final step of the proof involves applying a cylindrical transformation to equation (1). Once this is done, it can be seen that $g\left(u_{0}\right)=\bar{g}\left(u_{0}\right)$, and therefore $g\left(u_{0}\right) \geq 0$, as required.

\section{REFERENCES}

[B] K. Ball, Some remarks on the geometry of convex sets, Geometric Aspects of Functional Analysis (J. Lindenstrauss and V. D. Milman, eds.), Lecture Notes in Math., vol. 1317, Springer-Verlag, Berlin, 1988, pp. 224-231.

[Be] M. Berger, Convexity, Amer. Math. Monthly 97 (1990), 650-678.

[Bo] J. Bourgain, On the Busemann-Petty problem for perturbations of the ball, Geom. Funct. Anal. 1 (1991), 1-13.

[BL] J. Bourgain and J. Lindenstrauss, Projection bodies, Geometric Aspects of Functional Analysis (J. Lindenstrauss and V. D. Milman, eds.), Lecture Notes in Math., vol. 1317, SpringerVerlag, Berlin, 1988, pp. 250-270.

[BZ] Yu. D. Burago and V. A. Zalgaller, Geometric inequalities, Springer-Verlag, Berlin, 1988.

[B1] H. Busemann, Volume in terms of concurrent cross-sections, Pacific J. Math. 3 (1953), 1-12.

[B2] Wolumes and areas of cross-sections, Amer. Math. Monthly 67 (1960), 248-250; correction 67 (1960), 671.

[BP] H. Busemann and C. M. Petty, Problems on convex bodies, Math. Scand. 4 (1956), 88-94.

[CFG] H. T. Croft, K. J. Falconer, and R. K. Guy, Unsolved problems in geometry, Springer-Verlag, New York, 1991.

[ES] H. Edelsbrunner and S. S. Skiena, Probing convex bodies with X-rays, SIAM J. Comput. 17 (1988), 870-882.

[F] P. Funk, Über Flächen mit lauter geschlossenen geodätischen Linien, Math. Ann. 74 (1913), 278-300.

[G1] R. J. Gardner, Intersection bodies and the Busemann-Petty problem, Trans. Amer. Math. Soc. 342 (1994), 435-445 (to appear).

[G2] A positive answer to the Busemann-Petty problem in three dimensions, Ann. of Math. (2) (to appear).

[GV] R. J. Gardner and A. Volčič, Tomography of convex and star bodies, Adv. Math. (to appear). 
[Gi] A. A. Giannopoulos, A note on a problem of $H$. Busemann and C. M. Petty concerning sections of symmetric convex bodies, Mathematika 37 (1990), 239-244.

[Gie] M. Giertz, A note on a problem of Busemann, Math. Scand. 25 (1969), 145-148.

[GLW] P. R. Goodey, E. Lutwak, and W. Weil, Functional analytic characterizations of classes of convex bodies (to appear).

[GW] P. R. Goodey and W. Weil, Zonoids and generalizations, Handbook of Convex Geometry (P. M. Gruber and J. M. Wills, eds.), North-Holland, Amsterdam, 1993, pp. 1297-1326.

[GR] E. L. Grinberg and I. Rivin, Infinitesimal aspects of the Busemann-Petty problem, Bull. London Math. Soc. 22 (1990), 478-484.

[H] H. Hadwiger, Radialpotenzintegrale zentralsymmetrischer Rotationskörper und ungleichheitaussagen Busemannischer Art, Math. Scand. 23 (1968), 193-200.

[K] V. L. Klee, Ungelöstes Problem Nr. 44, Elem. Math. 17 (1962), 84.

[LR] D. G. Larman and C. A. Rogers, The existence of a centrally symmetric convex body with central sections that are unexpectedly small, Mathematika 22 (1975), 164-175.

[L] E. Lutwak, Intersection bodies and dual mixed volumes, Adv. Math. 71 (1988), 232-261.

[M] M. Meyer, On a problem of Busemann and Petty (to appear).

[MP] V. D. Milman and A. Pajor, Isotropic position and inertia ellipsoids and zonoids of the unit ball of a normed n-dimensional space, Geometric Aspects of Functional Analysis (J. Lindenstrauss and V. D. Milman, eds.), Lecture Notes in Math., vol. 1376, Springer-Verlag, Berlin, 1989, pp. 64-104.

[P] M. Papadimitrakis, On the Busemann-Petty problem about convex, centrally symmetric bodies in $\mathbb{R}^{n}$, Mathematika 39 (1992), 258-266.

[S] R. Schneider, Convex bodies: The Brunn-Minkowski Theory, Cambridge Univ. Press, Cambridge, 1993.

[SW] R. Schneider and W. Weil, Zonoids and related topics, Convexity and Its Applications (P. M. Gruber and J. M. Wills, eds.), Birkhäuser, Basel, 1983, pp. 296-317.

[T] S. Tanno, Central sections of centrally symmetric convex bodies, Kodai Math. J. 10 (1987), 343-361.

[W] W. Weil, Stereology: A survey for geometers, Convexity and Its Applications (P. M. Gruber and J. M. Wills, eds.), Birkhäuser, Basel, 1983, pp. 360-412.

[Z1] Gaoyong Zhang, Intersection bodies and the four-dimensional Busemann-Petty problem, Duke Math. J. 71 (1993), 223-240.

[Z2] Intersection bodies and the Busemann-Petty inequalities in $\mathbb{R}^{4}$, Ann. of Math. (2) (to appear).

Department of Mathematics, Western Washington University, Bellingham, WASHINGTON 98225-9063

E-mail address: gardner@baker . math . wwu . edu 